\title{
Analyzing SLE 88 memory management security using Interacting State Machines
}

\author{
David von Oheimb ${ }^{1}$, Volkmar Lotz ${ }^{1}$, Georg Walter ${ }^{2}$ \\ 1 Siemens AG, Corporate Technology, D-81730 Munich \\ e-mail: \{David.von.Oheimb/Volkmar.Lotz\}@siemens.com \\ 2 Infineon AG, Secure Mobile Solutions, D-81609 Munich \\ e-mail: Georg.Walter@infineon.com
}

The date of receipt and acceptance will be inserted by the editor

\begin{abstract}
The Infineon SLE 88 is a smart card processor that offers strong protection mechanisms. One of them is a memory management system, typically used for sandboxing application programs dynamically loaded on the chip. High-level (EAL5+) evaluation of the chip requires a formal security model.

We formally model the memory management system as an Interacting State Machine and prove, using Isabelle/HOL, that the associated security requirements are met. We demonstrate that our approach enables an adequate level of abstraction, which results in an efficient analysis, and points out potential pitfalls like non-injective address translation.
\end{abstract}

Keywords: Security, formal analysis, smart cards, memory management, Interacting State Machines, Isabelle/HOL.

\section{Introduction}

Since smart cards are becoming widely spread and are typically used for security-critical applications, smart card vendors face the demand for validating the security functionality of their cards wrt. adequacy and correctness. Third-party evaluation and certification is accepted as the appropriate approach, making it quite an active field. Certification of smart card processor products according to the Common Criteria [CC99] typically refers to the Smartcard IC Platform Protection Profile [AHIP01] and its augmentations like [AHIP02]. Based on these documents, the security target [WN03] for the Infineon SLE 88 smart card chip demands assurance level EAL5+ to be achieved, in particular requiring formal reasoning on the requirements level, viz. a formal security model. 
Infineon could make use of an extension of the established LKW model [LKW00] which already covers most aspects of security. Yet the SLE 88 offers a new security feature that requires special attention: a sophisticated memory management. For its evaluation we have developed a formal model which we describe in detail in the present article. The upcoming field of multi application smart cards motivate protection of applications from each other. The model shows that this can be effectively achieved with classical hardware based separation of memory areas. This feature may be used in particular within Java Virtual Machine implementations, yielding major progress in the area of dynamically loadable applications for smart cards.

The memory management security model is given in terms of Interacting State Machines (ISMs) introduced in [OL02]. ISMs are state-transition automata that communicate asynchronously on multiple input and output ports and thus can be seen as high-level Input/Output Automata [LT89]. They have turned out to be appropriate for the task of security modeling, for instance the full formalization of the LKW model with the Isabelle/HOL theorem prover [NPW02].

Most of related work on high EAL evaluation for smart cards, done e.g. at Trusted Logic and Philips, is unpublished. There are publications dealing with the Java Card runtime system [MT00] and with smart card operating systems in general $\left[\mathrm{SRS}^{+} 00\right]$. Note that these focus on software, while we focus on hardware.

\section{SLE 88 Memory Management}

In this section we introduce the virtual memory system of the SLE 88 with its associated security objectives and protection mechanisms. We start with a description of the security environment stating the processor's security objectives not pertaining to memory management and the security responsibilities of the smart card operating system and the applications.

\subsection{Security Environment}

The SLE88 is a smart card processor designed to support the protection of a smart card against threats including unauthorized access and modification of data as well as compromising the correct execution of the software residing on the card. From the security viewpoint, a smart card consists of the processor hardware, its $I C$ dedicated software, i.e., software providing a highlevel interface to hardware functions, and the smart card embedded software providing operating system (OS) and application functionality. The three layers share responsibility for achieving the desired level of security: the processor hardware achieves protection against tampering and side channel attacks, the IC dedicated software provides controlled access to hardwarebased security functions including encryption and memory management to 
the smart card embedded software, which in turn is in charge of enforcing the application-specific security policy.

The SLE88 comprises of the processor hardware and the IC dedicated software. Figure 1 gives an overview of its hardware structure, particularly emphasizing on the crypto co-processor used for memory and bus encryption. The hardware is designed to satisfy all security requirements imposed in [AHIP01]; its formal security model is given by the LKW model [LKW00].

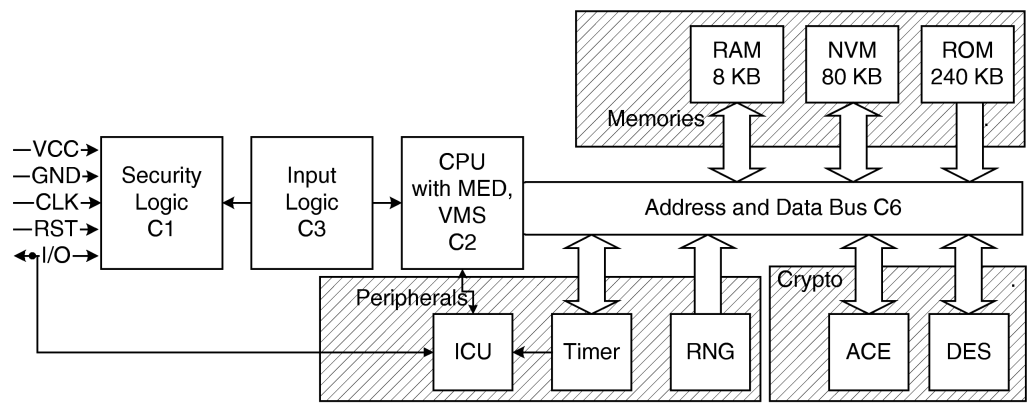

Fig. 1 SLE88 Block Diagram

This paper concentrates on the particular memory management security service offered through the IC dedicated software building upon the MMU (Memory Management Unit) hardware. It provides a virtual address space where access control attributes can be assigned to pages within that space. The attributes can be used by the smart card embedded software to define rules for information and control flow between applications. Note that the Memory Management Unit is only responsible for enforcing the protection according to the attributes set by the smart card embedded software. Thus, it does not define an application layer access control security policy on its own. The division of responsibility to achieve security between the smart card layers is reflected in the definition of the security requirements for memory management and is investigated in detail below.

\subsection{Memory Organization}

The physical memory of the SLE88 family is handled via 22 bit physical effective addresses (PEAs). Virtual memory is addressed via 32 bit virtual effective addresses (VEAs). The atomic units of the translation from virtual to physical addresses are pages of 64 bytes, which results in 6 bit wide displacements, i.e., address offsets. Peripheral hardware is memory mapped and thus can be accessed - and protected - in the same way as ordinary memory cells.

Typically, there are several independent software modules of different origin. Therefore, virtual memory is logically divided into 256 packages of 
equal size, such that the package address (PAD) makes up the upper 8 bits of the VEA. Packages 0 to 2 are privileged because they control security-critical entities. Package 0 contains the security layer (SL), package 1 contains the platform support layer (PSL), also known as hardware abstraction layer (HAL), and package 2 contains the operating system (OS). SL and PSL together form the IC dedicated software. Of the remaining regular packages, those with numbers 3 to 15 are reserved, while those with numbers 16 to 255 are available for (third-party) application software to be uploaded on demand. Figure 2 shows the structure of the address space in detail.

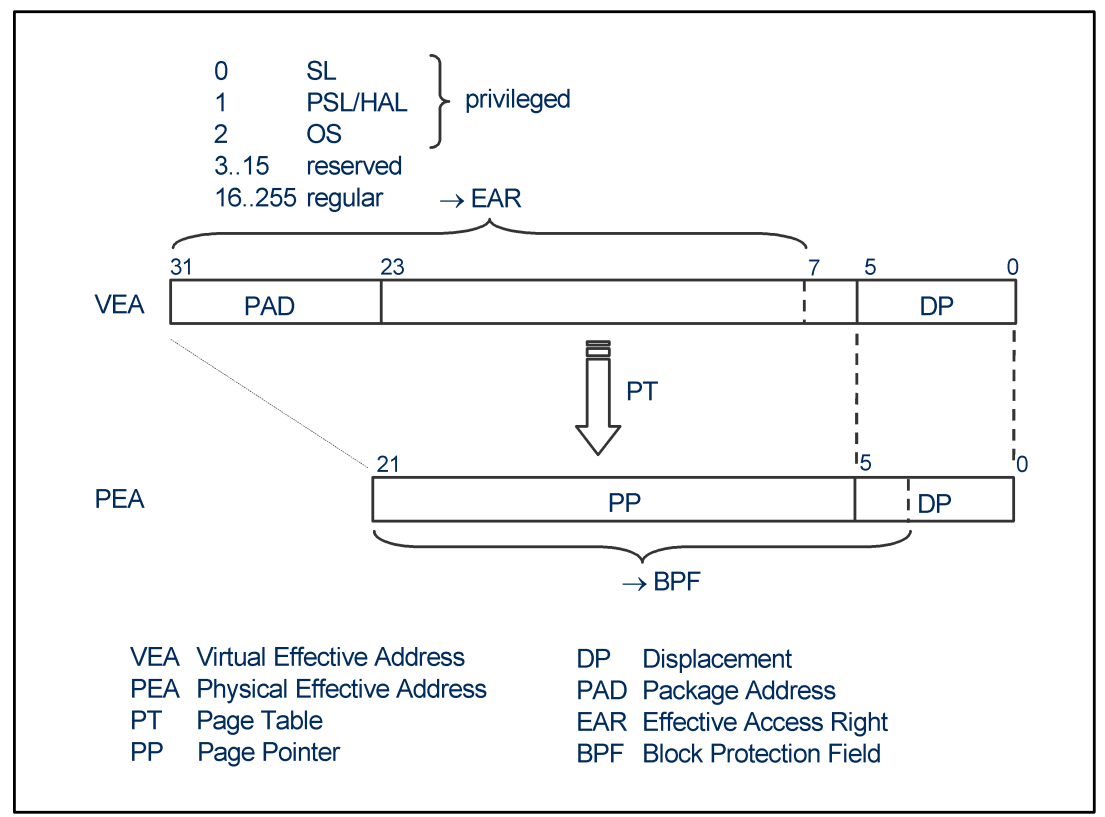

Fig. 2 SLE88 Address Structure

\subsection{Security Objectives}

The security objective relevant here is O.Mem-Access: "Area based Memory Access Control", defined in [WN03, §4.1]:

The TOE must provide the Smartcard Embedded Software with the capability to define restricted access memory areas. The TOE must then enforce the partitioning of such memory areas so that access of software to memory areas is controlled as required, for example, in a multi-application environment.

This means in particular that inter-package access to code and data should be restricted and that the corresponding protection attributes should be controlled by (specially protected) privileged packages only. Detail on 
the associated Security Functional Requirements (SFRs) may be found in $[\mathrm{WN} 03, \S 5.1 .1 .2]$.

Note that the requirement above does not refer to a particular access control security policy to be enforced on application level: rules may differ depending on the application domain (" partitioning ... is controlled as required") . The processor's task is therefore to provide the technical means to effectively support the definition and enforcement of application-specific access control policies rather than defining such policies on its own. Only parts of the policy are fixed and cannot be overwritten by attribute assignment of the smart card embedded software, namely those pertaining to SL and other privileged packages.

This is the reason why using classical access control or information flow models like Bell-LaPadula [BL73] and non-interference ${ }^{1}$ [GM82,Rus92] as well as integrity models like Biba [Bib77] and Clark-Wilson [CW87] (preserving integrity might also a motivation for requiring application separation) turned out to be inappropriate. Requirements imposed on policies, e.g., the domination relation being a lattice in Bell-LaPadula and Biba, and the separation-of-duty principle of Clark-Wilson, cannot be enforced on the processor level, since the processor is not intended to know about the particular application structure, their properties and protection needs. To formally model the requirement above, a security model had to be provided that concentrates on the processor's contribution to access control policy enforcement and reflects the division of responsibility between the processor and the smart card embedded software.

\subsection{Protection Mechanisms}

Virtual memory is associated with effective access rights (EARs) determining the read, write, and execute access of packages. Their granularity is 256 bytes, corresponding to the lower 8 bits of the VEAs. Moreover, each physical page block of 16 bytes, corresponding to the lower 4 bits of the PEA, is associated with additional security attributes referred to as block protection field $(B P F)$. The only information we will need in the model is a predicate called PASL specifying whether a page block should be accessible by SL only.

An EAR is given by a two-letter code where each of the letters may be W, R, X, or -, which specify read/write access to data, read-only access to data, executing access to code, and no access, respectively. The first letter refers to access within a package, while the second letter refers to access of one package (the source) to some other package (the target). The only allowed combinations are WW, WR, RR, W-, R-, and X-. Note that the EAR gives an implicit classification of memory sections as code or data. Code can be marked only with $\mathrm{X}$-, which indicates that inter-package code fetch

\footnotetext{
1 Note that, in general, non-interference properties can be well analyzed in our formal setting, see [Ohe04].
} 
is generally prohibited. Regardless of the EAR, privileged packages have free data access to all other packages except SL.

The meaning of the EARs can be described also with the following table.

\begin{tabular}{|c|c|c|c|c|}
\hline Source package: & \multicolumn{2}{|c|}{ Same as target } & \multicolumn{2}{|c|}{ Other than target } \\
\hline Target EAR & Read & Write & Read & Write \\
\hline WW & + & + & + & + \\
\hline WR & + & + & + & MPA/+ \\
\hline $\mathrm{RR}$ & + & MPA & + & MPA/+ \\
\hline W- & + & + & $\mathrm{MPA} /+$ & $\mathrm{MPA} /+$ \\
\hline$R-$ & + & MPA & $\mathrm{MPA} /+$ & $\mathrm{MPA} /+$ \\
\hline $\mathrm{X}-$ & MPA & MPA & MPA $/+$ & MPA/+ \\
\hline other & MPA & MPA & MPA & MPA \\
\hline
\end{tabular}

In the table, + means that the access is granted, and MPA means that a Memory Protection Alarm (MPA) is triggered. MPA $/+$ means + if the source is privileged and the target is not SL, and MPA in all other cases.

Apart from the restrictions on (linear) code fetch, inter-package control transfer is allowed only if the target holds a special PORT instruction sequence that defines the set of packages allowed to enter.

\subsection{Security Objectives Revisited}

Having introduced some of the details of the protection mechanisms available, we are now able to give a more detailed interpretation of the informal security requirements on memory management stated in the Security Target and introduced in 2.3. The properties to be achieved can be divided into two groups: enforcing separation of applications by controlling access to package memory areas as defined by the EAR setting according to the applications' security policies, and special protection of SL being responsible for managing the security attributes.

Pertaining to application separation, we require:

- Read/write/execute accesses have to respect the given EAR settings, i.e., only those access requests should be granted that are allowed by the actual EARs.

- Inter-package control transfer is only allowed through PORT instructions.

The complexity in arguing about these properties is introduced through the possibility of non-injective address mappings and the functionality to modify the page table and the EAR settings itself. Non-injective mappings assigning a page to multiple virtual addresses with different EAR assignments are critical because the memory management does not offer conflict resolution strategies but still should avoid weakening access control restrictions by exploiting different access paths to physical addresses. 
With respect to protection of SL, we require:

- Memory areas assigned to SL can only be changed by SL itself, i.e., the integrity of the security functionality is maintained. This also covers protection against buffer overflow attacks on SL.

- SL can only be accessed in a controlled way, namely through the PSL package. Thus, PSL can be used to apply specific filtering of attribute modification requests by the smart card embedded software, which allows to implement parts of an application separation policy on the level of the IC dedicated software.

Note that protection of SL does not require PSL to be trustworthy. However, SL protection will rely on benign behaviour of SL itself and appropriate initial EAR settings.

\section{Formalism and Tools}

For modeling (and partially verifying) the SLE 88 memory management, we take the ISM approach [OL02]. This means that we formally define and analyze its security model as an Interacting State Machine (ISM) [Ohe02] within the theorem prover Isabelle/HOL [NPW02]. Though the model described in this article does not actually make use of parallel composition of ISMs and related concepts, we give the full definitions here for completeness. For further advances to the ISM formalism, namely generic ISMs and their instantiations to dynamic ISMs and ambient ISMs, see [OL03, KO03].

\subsection{Concept of Interacting State Machines}

An Interacting State Machine (ISM) is an automaton whose state transitions may involve multiple input and output simultaneously on any number of ports. As the name suggests, the key concepts of ISMs are states (and in particular the transitions between them) and interaction. By interaction we mean explicit buffered communication via named ports (which are also called connections), where on each port, (typically) one receiver listens to possibly many senders.

Any number of ISMs may be composed in parallel by interleaving their transitions and forming I/O connections among peer ISMs. The local state of the resulting ISM is essentially the Cartesian product of the local states of its components. The top-level composition is called an ISM system. In [OL03] we extend the ISM concept by the notions of global state and commands that may affect the global state.

A configuration of an ISM consists of its input buffer state and local state. The local state may have arbitrary structure but typically is the Cartesian product of a control state which is of finite type and a data state which is a record of named fields representing local variables. Each ISM has a single ${ }^{2}$ local initial state.

\footnotetext{
2 If a non-singleton set of initial states is required, this may be simulated by nondeterministic spontaneous transitions from a single dummy initial state.
} 


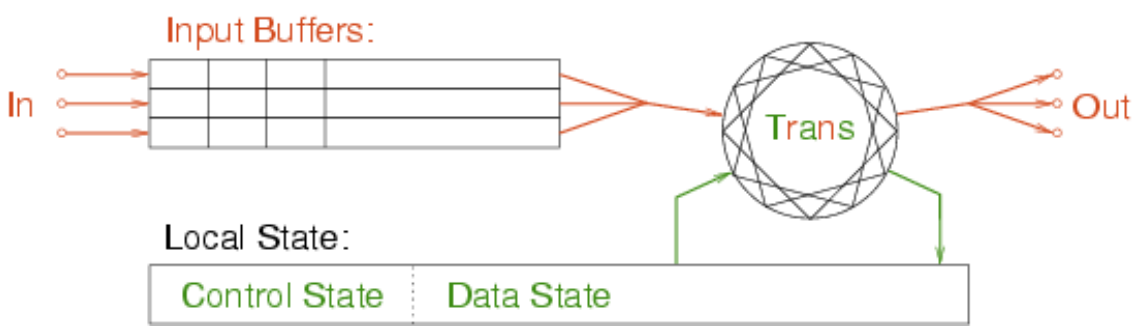

Fig. 3 ISM structure

The input buffers of an ISM are a family of (unbounded) message FIFOs, indexed by port names. The buffers are not actually part of an ISM but exist merely as intermediate data structures within parallel composition during ISM runs. Input buffers can (but in most applications should not) be shared among ISMs, which leads to competition on the input without fairness constraints.

Message exchange is triggered by an output operation of any ISM within the system. Input from the environment may be modeled with suitable ISMs. Inputs cannot be blocked, i.e. they may occur at any time, appending the received value to the corresponding FIFO. Values stored in the input buffers related to an ISM are received and processed by the ISM when it is ready to do so.

The actions of ISMs are given as user-defined transitions, which may be nondeterministic and can be specified in any relational style. Thus for each transition the user has the choice to define it in an operational (i.e., executable) or axiomatic (i.e., property-oriented) fashion or a mixture of the two. Transition rules specify that - potentially under some precondition that typically includes matching of messages in the input buffers - the ISM consumes some input, makes a local state transition, and produces some output. The output is appended to the respective input buffers specified by port names. Direct or indirect feedback is possible. Multicast is not directly supported but may be explicitly modeled easily.

An ISM system run is any prefix of the sequence of configurations reachable from the initial configuration. The length of a run is not bounded but finite. Finiteness allows for a simple trace semantics, but on the other hand implies that we cannot handle liveness properties. Yet we do not feel this as a real restriction because most relevant properties are essentially safety properties: practical guarantees about the existence of future events typically involve timeouts.

Transitions of different ISMs that are composed in parallel cannot directly interfere with each other but are related only by the causality wrt. the messages interchanged. Execution gets stuck (i.e., deadlocks) when there is no component that can perform any step. As is typical for reactive systems, there is no built-in notion of final or accepting states. 


\subsection{ISM Semantics}

This subsection gives the logical meaning of ISMs, which is both an extension and a slight simplification of the definitions given in [Ohe02]. It may be skipped for a first reading of this article.

First some general remarks on the presentation: all definitions and proofs have been developed as a hierarchy of Isabelle/HOL theories and machinechecked using this tool. One important effect of this approach is that many kinds of mistakes like type mismatches can be ruled out. Using the $\mathrm{HT}_{\mathrm{E}} \mathrm{X}$ documentation feature of Isabelle would even preclude typographic slips in the presentation but on the other hand would introduce some technicalities many readers would not be familiar with. Therefore, we give the semantics in traditional "mathematical" style in order to enhance readability. We sometimes make use of $\lambda$-abstraction borrowed from the $\lambda$-calculus, but write (multi-argument) function application in the conventional form, e.g. $f(a, b, c)$. Occasionally we make use of partial application (aka. currying), such that, in the example just given, $f(a, b)$ is an intermediate function that requires a third parameter before yielding the actual function result.

In order to help understanding the abstract formal definitions, we give a simple running example. It describes two producers sending random integer values to a port named Inlet of a consumer which sums them up in a local variable named $A c c u$ initialized to 0 . The system can be depicted as

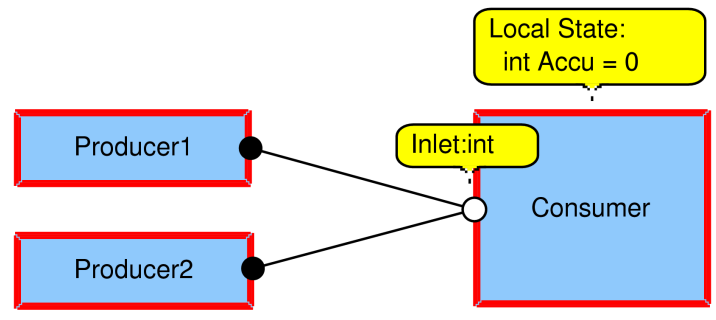

Fig. 4 Producer-Consumer example

3.2.1 Message Families Let $\mathcal{M}$ be the type of all messages potentially exchanged by ISMs and $\mathcal{P}$ the type of port names. Then the message families, which are used to denote both input ${ }^{3}$ buffers and input/output patterns, have type $M S G s=\mathcal{P} \rightarrow \mathcal{M}^{*}$ where $\mathcal{M}^{*}$ is any finite sequence of elements of $\mathcal{M}$. We will make use of the following operations on message families:

- the term $\not \subset$ denotes the empty message family $\lambda p$. \langle\rangle where \langle\rangle denotes the empty sequence

- the term $m \operatorname{dom}(m)$ abbreviates $\{p \cdot m(p) \neq\langle\rangle\}$, i.e. the domain of $m$

- the infix operation.@. concatenates two message families $m$ and $n$ pointwise: $(m$.@. $n)(p)=m(p) @ n(p)$

\footnotetext{
${ }^{3}$ Recall that output buffers are not required.
} 
In our example, $\mathcal{P}=\{$ Inlet $\}$ and $\mathcal{M}=$ int, such that $M S G s=\{$ Inlet $\} \rightarrow$ int ${ }^{*}$. Assuming $m=\lambda p$. if $p=$ Inlet then $\langle 1,-3\rangle$ else \langle\rangle , which can be written also as $m=\not Q \not($ Inlet $:=\langle 1,-3\rangle)$, and $n=\not Q($ Inlet $:=\langle 6\rangle)$, one obtains $m \operatorname{dom}(m)=\{$ Inlet $\}$ and $m$.@. $n=\not{C}($ Inlet $:=\langle 1,-3,6\rangle)$.

3.2.2 States and Transitions A set of ISM transitions has type TRANS $(\Sigma)$ $=\wp((M S G s \times \Sigma) \times(M S G s \times \Sigma))$ where the parameter $\Sigma$ stands for the type of the local state and the two occurrences of MSGs stand for input and output patterns, respectively. For the consumer in the above example, $\Sigma=$ int. Each element has the form $\left((i, \sigma),\left(o, \sigma^{\prime}\right)\right)$ and means that the ISM can (possibly nondeterministically) perform a step from local state $\sigma$ to $\sigma^{\prime}$, consuming input $i$ and producing output $o$. Simultaneous input and/or output on multiple channels can be specified because both $i$ and $o$ each denote whole message families. In contrast to the original definition of ISMs [Ohe02], within a transition, input is described by patterns of messages consumed in the given step - not by a transition between the state of the input buffer before and after the transition. This simplifies the definition of single ISMs and shifts the concept of input buffering to the places where it is indispensable: at the definitions of parallel composition and automata runs.

3.2.3 Elementary ISMs An ISM is given as a quadruple ${ }^{4} a=(\operatorname{In}(a)$, Out $(a)$, $\sigma_{0}(a)$, Trans $\left.(a)\right)$ of type $\operatorname{ISM}(\Sigma)=\wp(\mathcal{P}) \times \wp(\mathcal{P}) \times \Sigma \times \operatorname{TRANS}(\Sigma)$ where

- $\operatorname{In}(a)$ is the set of input port names

- Out $(a)$ is the set of output port names

- $\sigma_{0}(a)$ is the initial local state

- $\operatorname{Trans}(a)$ is the transition relation

Such an ISM is well-formed iff all the port names actually used in the transitions for input or output respect the I/O interface of the ISM, i.e. $\operatorname{ipns}(a) \subseteq \operatorname{In}(a)$ and $\operatorname{opns}(a) \subseteq \operatorname{Out}(a)$ where

- ipns $(a)=\bigcup_{t \in \operatorname{Trans}(a)} \operatorname{mdom}\left(\left(\lambda\left((i, \sigma),\left(o, \sigma^{\prime}\right)\right) \cdot i\right)(t)\right)$

- opns $(a)=\bigcup_{t \in \operatorname{Trans}(a)} \operatorname{mdom}\left(\left(\lambda\left((i, \sigma),\left(o, \sigma^{\prime}\right)\right) . o\right)(t)\right)$

Note that $\operatorname{In}(a)$ and $\operatorname{Out}(a)$ may overlap, which allows for direct feedback within parallel composition.

In the above example, the consumer ISM can be given as Consumer $=$ $(\{$ Inlet $\}, \emptyset, 0,\{(\mathscr{Q}($ Inlet $:=\langle n\rangle), a, \not, a+n) \mid n \in$ int $\wedge a \in$ int $\})$. This ISM is well-formed since ipns $($ Consumer $)=\{$ Inlet $\}$ and opns $($ Consumer $)=\emptyset$. The producers don't need local variables, so we give their local state the dummy type unit with sole element $\bullet$. They can be defined as Producer $_{i}=$ $(\emptyset,\{$ Inlet $\}, \bullet,\{(\mathscr{Q}, \bullet, \mathscr{Q}($ Inlet $:=\langle n\rangle), \bullet) \mid n \in$ int $\})$ for $i=1,2$.

\footnotetext{
4 The definition pattern $x=\left(\operatorname{sel}_{1}(x), \operatorname{sel}_{2}(x), \ldots\right)$ should not be understood as a recursive definition of $x$ but as a shorthand introducing a tuple with typical name $x$ and with selectors (i.e., projection functions) $s l_{1}, s_{2} l_{2}, \ldots$
} 
3.2.4 Runs Below we will define composite ISM runs, i.e. the parallel composition and execution of a family of ISMs, directly in one step. Nevertheless, we first define the two notions of ISM runs and parallel composition independently. Defining parallel composition in isolation not only makes it easier to understand but also enables hierarchical analysis and design.

The open runs of an ISM $a$, denoted by $\operatorname{Runs}(a) \in \wp\left(\Sigma^{*}\right)$, are finite sequences of states that are inductively defined as

$$
\begin{aligned}
& \overline{\left\langle\sigma_{0}(a)\right\rangle \in \operatorname{Runs}(a)} \\
& s s \frown \sigma \in \operatorname{Runs}(a) \\
& \frac{\left((i, \sigma),\left(o, \sigma^{\prime}\right)\right) \in \operatorname{Trans}(a)}{s s^{\frown} \sigma^{\frown} \sigma^{\prime} \in \operatorname{Runs}(a)}
\end{aligned}
$$

The operator $\frown$ appends elements to a sequence.

This form of runs is called open because in each step the environment provides arbitrary input to the ISM, and any output of the ISM is discarded. If feedback from output to input is desired, one can achieve this by applying the parallel composition operator to the singleton family of ISMs consisting just of $a$, described next.

In the example, an open run of the consumer is any sequence of integers starting with 0 , for instance $\langle 0,1,-2\rangle$ if the input happens to be 1 and -3 .

3.2.5 Parallel Composition Any number of ISMs can be combined in parallel to form a single composite ISM, which may be further combined with others, etc.

The parallel composition $\|_{i \in I} A_{i}$ of a family of ISMs $A=\left(A_{i}\right)_{i \in I}$ is an ISM of type $I S M\left(C O N F\left(\Pi_{i \in I} \Sigma_{i}\right)\right)$ where $I$ is any index set $I$ and for any $X$, the type of an ISM configuration $\operatorname{CONF}(X)$ is defined as $M S G s \times X$. Here $M S G s$ stands for the type of internal buffers. The composite ISM is defined as the quadruple $\left(\operatorname{AllIn}(A) \backslash \operatorname{AllOut}(A), \operatorname{AllOut}(A) \backslash \operatorname{AllIn}(A),\left(\not, S_{0}(A)\right)\right.$, $\operatorname{PTrans}(A))$ where

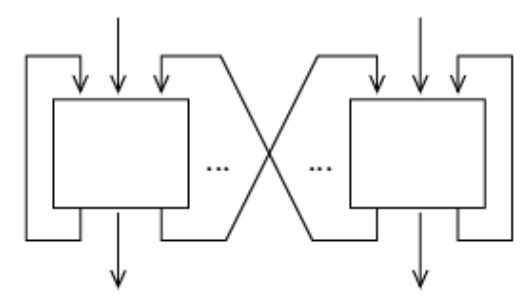

Fig. 5 General pattern of feedback within parallel composition

- $\operatorname{AllIn}(A)=\bigcup_{i \in I} \operatorname{In}\left(A_{i}\right)$

- $\operatorname{AllOut}(A)=\bigcup_{i \in I} \operatorname{Out}\left(A_{i}\right)$

- $\not$ gives the initial value of the internal buffers, which are used to handle I/O among peers as well as direct feedback

- $S_{0}(A)=\Pi_{i \in I}\left(\sigma_{0}\left(A_{i}\right)\right)$ is the Cartesian product of all initial local states 
- PTrans $(A)$ of type $\operatorname{TRANS}\left(\operatorname{CONF}\left(\Pi_{i \in I} \Sigma_{i}\right)\right)$ is the parallel composition of their transition relations.

In our example, we combine the two producers and the consumer in parallel. Let $I=\{1,2,3\}$ and $A_{1}=$ Producer $_{1}, A_{2}=$ Producer $_{2}$, and $A_{3}=$ Consumer $\}$. Then $\Pi_{i \in I} \Sigma_{i}=$ unit $\times$ unit $\times$ int and the composite ISM $A$ is $(\emptyset, \emptyset,(\mathscr{Q},(\bullet, \bullet, 0)), \operatorname{PTrans}(A))$ because $\operatorname{AllIn}(A)=\operatorname{AllOut}(A)=\{$ Inlet $\}$.

The pre- and post-states in the composed transition relation refer not only to the Cartesian product of all local states but also to a message family $b$. As already mentioned above for the initial state, the role of $b$ is to buffer internal I/O. Apart from this, the composed transition relation is defined simply as the interleaving of the transitions of the component ISMs:

$$
\begin{gathered}
j \in I \\
\frac{\left((i, \sigma),\left(o, \sigma^{\prime}\right)\right) \in \operatorname{Trans}\left(A_{j}\right)}{\left(\left(i_{\mid \overline{\operatorname{AllOut}(A)},},\left(i_{\mid \operatorname{AllOut}(A)} @ @ . b, S[j:=\sigma]\right)\right),\right.} \\
\left.\left(o_{\mid \overline{\operatorname{AllIn}(A)}}, \quad\left(b . @ . O_{\mid \operatorname{AllIn}(A)}, S\left[j:=\sigma^{\prime}\right]\right)\right)\right) \in \operatorname{PTrans}(A)
\end{gathered}
$$

where

$-S[j:=\sigma]$ denotes the replacement of the $j$-th component of the tuple $S$ by $\sigma$

- $m_{\mid P}$ denotes the restriction $\lambda p$. if $p \in P$ then $m(p)$ else \langle\rangle of the message family $m$ to the set of ports $P$

$-i_{\mid \overline{A l l O u t(A)}}$ denotes those parts of the input $i$ provided not by the output of peer ISMS but by outer ISMs

$-i_{\mid A l l O u t(A)}$ denotes the internal input from peer ISMs or direct feedback, which is taken from the current buffer contents $b$

- $o_{\mid} \overline{\operatorname{AllIn}(A)}$ denotes those parts of the output $o$ provided to outer ISMs

- $o_{\mid} \operatorname{Allin}(A)$ denotes the internal output to peer ISMs or direct feedback, which is added to the current buffer contents $b$

In the example,

$$
\begin{aligned}
& \operatorname{PTrans}(A)=\{(\not \subset,(b,(\bullet, \bullet, a)), \not{Q},(b \text {.@. } \mathscr{Q}(\text { Inlet }:=\langle n\rangle),(\bullet, \bullet, a))) \mid \\
& n, a \in \text { int } \wedge a \in \text { int } \wedge b \in M S G s\} \\
& \cup\{(\mathscr{O},(\mathscr{Q}(\text { Inlet }:=\langle n\rangle) \text { @@. b, }(\bullet, \bullet, a)), \mathscr{Q},(b,(\bullet, \bullet, a+n))) \mid \\
& n, a \in \text { int } \wedge a \in \text { int } \wedge b \in M S G s\} \text {. }
\end{aligned}
$$

The first part of the union describes the effect of a transition of either producer: a random value $n$ is appended to the internal buffer $b$ (Inlet). The second part describes that the consumer takes one such value out of the buffer and adds the value to its local accumulator variable.

A possible run of the example system, i.e. a member of $\operatorname{Runs}(A)$, is $\langle(\mathscr{Q},(\bullet, \bullet, 0)), \quad(\mathscr{Q}($ Inlet $:=\langle 1\rangle,(\bullet, \bullet, 0)), \quad(\mathscr{Q}($ Inlet $:=\langle 1,-3\rangle,(\bullet, \bullet, 0))$, $(\mathscr{Q}($ Inlet $:=\langle-3\rangle,(\bullet, \bullet, 1)), \quad(\not,,(\bullet, \bullet,-2)), \quad(\mathscr{Q}($ Inlet $:=\langle 6\rangle,(\bullet, \bullet,-2))$, $(,,(\bullet, \bullet, 4))\rangle$ where first 1 and -3 are produced (by either of the producers), then both values get consumed, then 6 is produced and immediately thereafter consumed, such that the final local state of the consumer is $0+1-3+6=4$. 
A parallel composition is well-formed iff the inputs of the individual components do not overlap: $\forall i j . i \neq j \longrightarrow \operatorname{In}\left(A_{i}\right) \cap \operatorname{In}\left(A_{j}\right)=\emptyset$. On the other hand, outputs may overlap, which allows the outputs of different ISMs to interleave nondeterministically. This is the case for our example system: as can be seen easily, input ports do not overlap but outputs do.

A family $A$ of ISMs is called closed iff $\operatorname{AllIn}(A)=\operatorname{AllOut}(A)$, i.e. there is no interaction with any outside ISMs. Our example system is closed. If a system is modeled with a closed ISM family and input from the environment is important, this may be modeled with an ISM that belongs to the family and does nothing but generating all possible input patterns.

When composing ISMs, it is occasionally necessary to prevent name clashes or to hide connections, which can be achieved by suitable renaming of ports.

3.2.6 Composite Runs We define ISM runs not only for single (possibly composite) ISMs but also directly for closed families of ISMs intended to run in parallel. The above definition of parallel composition may be used in combination with composite runs to describe inner (possibly nested) levels of parallel composition.

The set of all possible composite runs is denoted by $\operatorname{CRuns}(A)$ and has type $\wp\left(\left(\operatorname{CONF}\left(\Pi_{i \in I} \Sigma_{i}\right)\right)^{*}\right)$ corresponding to the ISM type $\operatorname{ISM}\left(\Pi_{i \in I} \Sigma_{i}\right)$. Its elements are finite sequences of configurations, inductively defined as

$$
\begin{gathered}
\overline{\left\langle\left(\not, S_{0}(A)\right)\right\rangle \in \operatorname{CRuns}(A)} \\
j \in I \\
c s \frown(i . @ . b, S[j:=\sigma]) \in \operatorname{CRuns}(A) \\
\left((i, \sigma),\left(o, \sigma^{\prime}\right)\right) \in \operatorname{Trans}\left(A_{j}\right) \\
\overline{c s \frown(i . @ . b, S[j:=\sigma]) \frown\left(b . @ . o,\left(S\left[j:=\sigma^{\prime}\right]\right)\right) \in \operatorname{CRuns}(A)}
\end{gathered}
$$

Traces of composite runs have the form $\left\langle\left(\not, S_{0}(A)\right),\left(b_{1}, S_{1}\right),\left(b_{2}, S_{2}\right), \ldots\right\rangle$ where each element of the sequence is a pair of the current internal buffer contents and the Cartesian product of all the currently relevant local states.

One can show that composite runs of any closed family $A$ of well-formed ISMs are equivalent to the runs of the parallel composition of the same family: wf_isms $(A) \wedge \operatorname{closed}(A) \longrightarrow \operatorname{Runs}\left(\|_{i \in I} A_{i}\right)=\operatorname{CRuns}(A)$. Of course, this is the case for our running example since the system is closed.

\subsection{Isabelle/HOL Representation}

When aiming at rigorous formal modeling or even system verification, tools performing syntactic checks, type checks, and mechanized proofs are essential. We employ the theorem proving system Isabelle/HOL because of excellent experience with this tool.

Isabelle [NPW02] is a generic interactive theorem prover that has been instantiated to many logics, in particular the very practical Higher-Order 
Logic (HOL). Despite of one nuisance ${ }^{5}$, we consider Isabelle/HOL the most flexible and mature modeling and verification environment available. Using it, system properties can be expressed easily and adequately and can be verified using powerful proof methods. Furthermore, Isabelle offers good facilities for textual presentation and documentation.

ISMs can be defined in special Isabelle theory sections. Their standard interpretation is the meta theory described in $\S 3.2$. It is implemented by an Isabelle plug-in [Nan02] in connection with a library of Isabelle theories. ISMs can also be defined graphically using the CASE tool AutoFocus [HSSS96] and then translated to the Isabelle/HOL representation using a tool program [Nan02].

An ISM section is introduced by the keyword ism and has the following general structure ${ }^{6}$ :

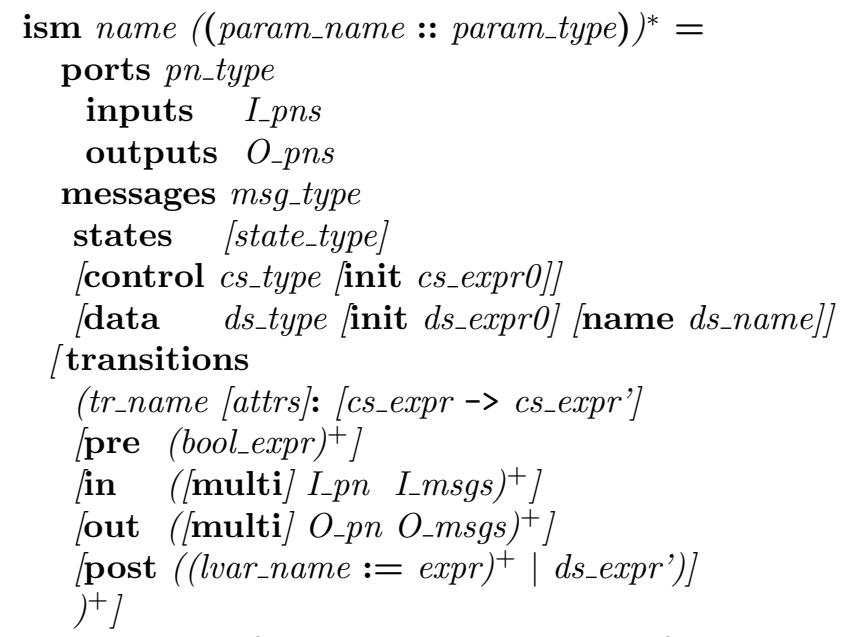

The meaning of the individual parts is as follows.

- The ISM definition will be referred to by name. It may have any number of parameters, each declared by param_name and a corresponding param_type. The parameters may be used throughout the definition body.

- The type expression pn_type gives the Isabelle/HOL type of the port names, while $I_{-} p n s$ and $O_{-}$pns denote the set of input and output port names, respectively.

- The type expression msg_type gives the type of the messages, which is typically an algebraic datatype with a constructor for each kind of message.

${ }^{5}$ The only drawback of Isabelle/HOL for applications like ours is the lack of dependent types: for each system modeled there is a single type of message contents into which all message data has to be injected, and the same holds for the local ISM states. The alternative prover PVS supports dependent types, but on the other hand it is less flexible, in particular, user-defined theory sections are not possible.

6 [...] marks optional parts, $(\ldots)^{+}$means one or more comma-delimited occurrences 
- The optional state_type should be given if the current ISM forms part of a parallel composition and the state types of the ISMs involved differ. In this case, state_type should be a free algebraic datatype with a constructor for each state type of the ISMs involved.

The type expressions cs_type and ds_type give the types of the control and data state, respectively, while the optional terms $c s_{-}$exprO and ds_expr0 specify their initial values - if not given, they default to some arbitrary value. Either (i.e., not both) the control state or the data state may be absent.

The optional logical variable name $d s_{-} n a m e$, which defaults to $s$, may be used to refer to the whole data state within transition rules.

Transitions are given via named rules where attrs is an optional list of attributes, e.g. [intro]. The control states (if any) before and after the transition are specified by the expressions ${ }^{7}$ cs_expr and cs_expr'.

Expressions within a rule may refer to the logical data state variable mentioned above. In particular, assuming that $s$ is the name of the data state variable, then the value of any local variable I var of the ISM may be referred to by lvar $s$. The scope of free variables appearing in a rule is the whole rule, i.e. free variables are implicitly universally quantified (immediately) outside each rule.

All the following parts of a transition rule are optional:

- The pre part contains guard expressions bool_expr, i.e. preconditions constraining the enabledness of a transition.

- The in part gives input port names (or sets of them if preceded by multi) $I_{-} p n$, each in conjunction with a list $I_{-} m s g s$ of message patterns expected to be present in the corresponding input buffer(s). When an ISM executes a transition, any free variables in message patterns are bound to the actual values that have been input. Each port names should appear at most once within a in part. Any input port not explicitly mentioned is left untouched.

- The out part gives output port names $O_{-} p n$, each in conjunction with an expression $O_{-} m s g s$ denoting a list of values designated for output to the corresponding port. The variant using multi is used to specify multicasts. Each port name should be used at most once within each out part. Any output port not mentioned does not obtain new output.

- The post part describes assignments of values expr to the local variables lvar_name of the data state. Variables not mentioned remain invariant. Alternatively, an expression $d s_{-}$expr' may be given that represents the entire new data state after the transition. Assignments to the local variables suit an operational style, whereas an axiomatic style can be achieved using $d s_{-}$expr' (in conjunction with suitable constraints in the preconditions).

\footnotetext{
7 These need not be constant but may contain also variables, which is useful for modeling generic transitions. In this case, one such transition has to be represented by a set of transitions within AutoFocus.
} 
An ism theory section is translated to Isabelle/HOL concepts in a straightforward way using an extension to Isabelle, as described in [Nan02]. In particular, each ISM section is translated to a record definition with the appropriate fields, the most complex one being the transition relation, which is defined via an inductive (but not actually recursive) definition.

The meta theory of ISMs that we have defined in Isabelle/HOL includes all concepts mentioned in $\S 3.2$, in particular well-formedness, renaming, parallel composition, runs, and composite runs. Further auxiliary concepts are introduced as well, in particular reachability and induction schemes related to ISM runs. The characteristic properties of these concepts, as required for system verification, are derived within Isabelle/HOL. All details of the meta theory may be found in [ON02].

\section{System Model}

In order to provide an comprehensive instructive presentation of the formal model, we reproduce all the definitions, lemmas and theorems (essentially leaving out proof scripts and a few other parts needed for technical reasons only), augmented with comments, just as they appear in the Isabelle theory sources ${ }^{8}$. By employing the automatic LTEX typesetting facility of Isabelle, we achieve on the one hand maximal accuracy of the presentation, retaining the mathematical rigor and the "flavor" 9 of the machine-checked specifications, and on the other hand good readability by using standard logical notation as far as possible and interspersing textual explanations and motivation.

A very important design principle is to keep a high level of abstraction, which improves readability and simplifies the proofs. Therefore, we model only those features that are strictly relevant for security, abstracting away unnecessary detail caused e.g. by efficiency optimizations. For the same reason we often use a modeling technique called underspecification, i.e. for part of the logical types and constants we do not give full definitions but only declarations of their names.

\subsection{Overview}

Following the standard approach to security analysis, we provide a system model describing the abstract behavior of the memory management and formalize the security objectives as properties of the system model. The state-based ISM approach fits well with modeling both the page table and the physical memory as state components of the system, mapping virtual

\footnotetext{
8 Isabelle/HOL adopts the notational standards of functional programming, writing for instance (multi-argument) function application as $f \times \mathrm{y}_{z}$ instead of $f(x, y, z)$.

9 For example, the order of definitions is strictly bottom-up.
} 
addresses to physical addresses and physical addresses to values. Our specification of (both virtual and physical) addresses represents the structure of the memory organization as described in $\S 2.2$. Values are only of interest in case of PORT instructions; we leave other values unspecified. The model further includes mappings describing the assignments of BPFs to page blocks and EARs to sections of the virtual address space.

To complete the system model, state transitions represent the different kinds of memory access that may occur. For each of them there is a corresponding input message for the ISM triggering a transition. Each transition produces an output stating whether the access is granted or denied. In case of denial, we have different output messages representing the different traps or alarms. The computation of the output refers to our formalization of the protection rules stated in $\S 2.4$. A transition may also result in modifications of state components, for instance, write access to the main memory or page table updates.

\subsection{Addressing}

First we have to define several aspects of the SLE 88 address space introduced in $\S 2.2$. These include the type of package addresses, $P A D$, defined as the disjoint sum of privileged and regular PADs, where we enumerate all three possibilities for privileged packages but do not specify the actual range of regular PADs:

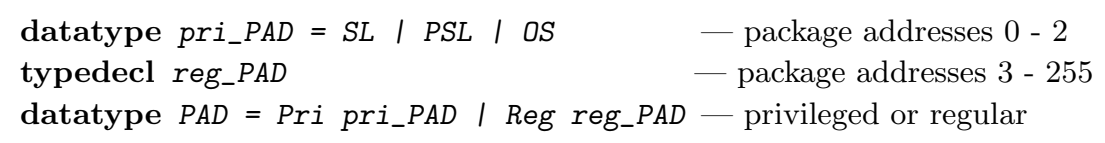

Next, we define a predicate distinguishing privileged from regular packages.

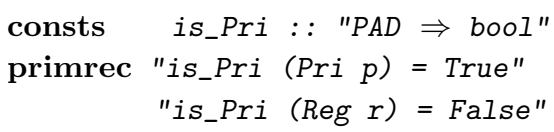

While PADs form the upper (i.e., most significant) part of virtual addresses, displacements $D P$ form the lower sections used for addressing individual bytes of memory within a page. We need to split them further because there are four page blocks within a page that are associated with their own BPFs. Note that despite the names that contain numbers giving bit positions, we do not actually specify the concrete ranges of the types declared but just state that $D P$ is the Cartesian product of the two other types:

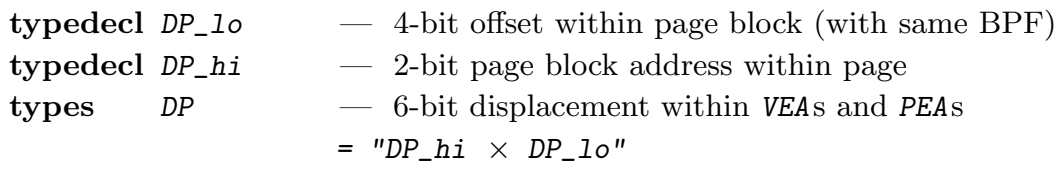


A virtual effective address consists of the package address, a middle part that we call VEA_mid, and the displacement. We have to further split the middle part because only the upper 16 bits of it are used to determine the EAR associated with the address. We also define the type VP of virtual page pointers which will be mapped to physical page pointers.

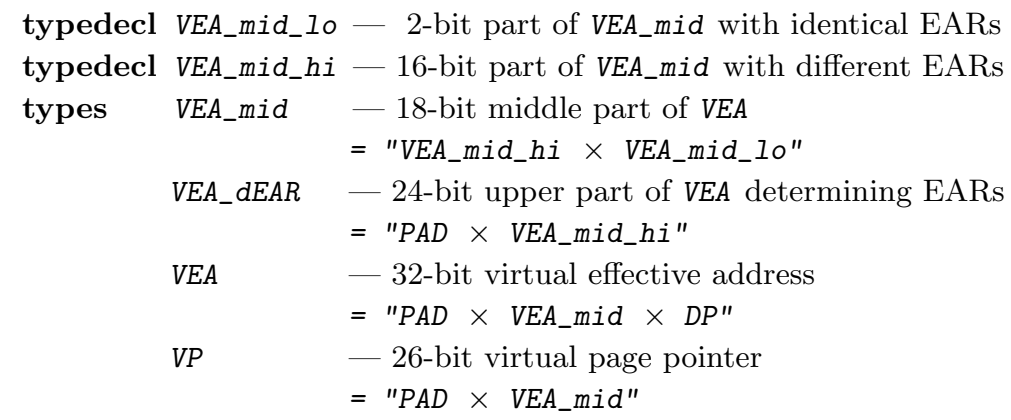

Physical page pointers $P P$ are combined with displacements to form physical effective addresses. The part of PEAs determining the BPF is called $P E A_{-} d B P F$.

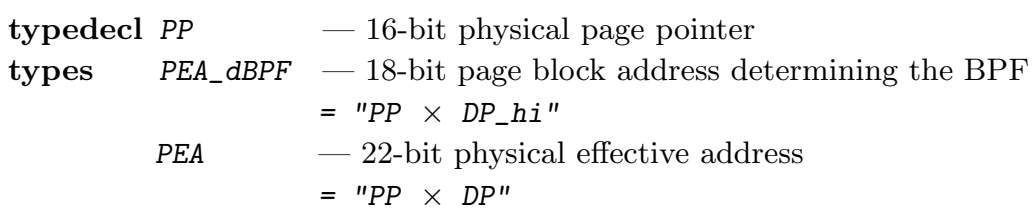

We define an auxiliary function $P A D$ extracting the package information from any address containing a PAD as its uppermost part, simply by projecting on this first part of the tuple: $P A D(p a d, \mathrm{x})=$ pad

\subsection{Effective Access Rights}

We enumerate all allowed EARs as defined in $§ 2.4$ and relate them with the access that they grant by functions for intra-package and inter-package access.

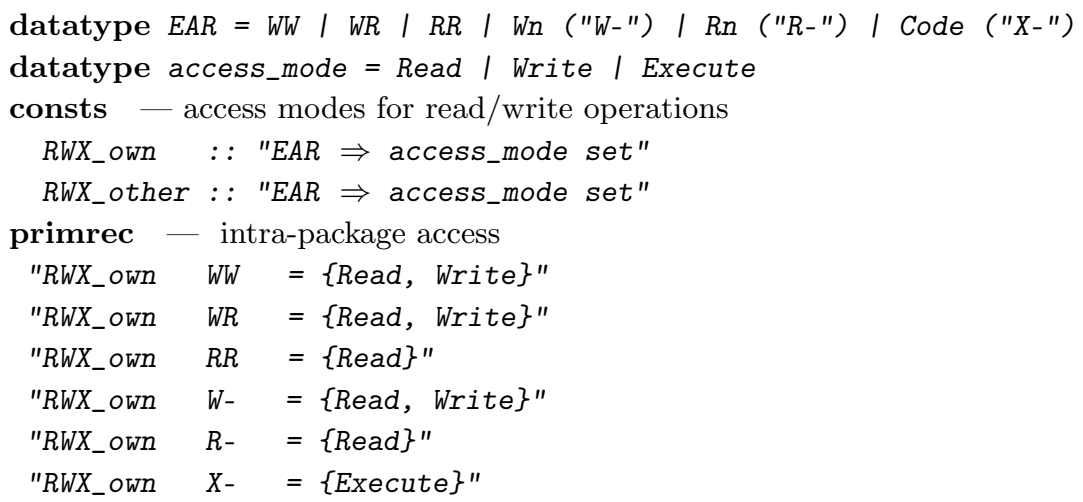




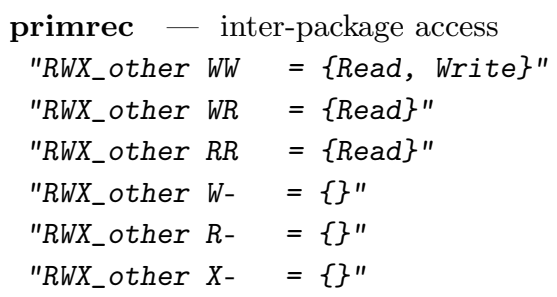

\section{$4 \cdot 4$ State}

Our abstract model of the SLE 88 memory management state consists of three aspects that are crucial for the security analysis:

- the physical memory contents (where the only sort of value we are interested in is a PORT instruction sequence with its associated set of packages) and the PASL predicate associated with page blocks

- the essentials of the page table entries, i.e. page mapping and EARs - there is no need for us to model complex structures like translation lookaside buffers and multi-level page tables required merely for optimization

- the package information contained in the current program counter and in the return address stack

For simplicity, we model PORT instructions as atomic values. We define them as one of the alternatives in a (free) datatype, which implies that they can be distinguished from all other instructions. This is adequate because the SLE 88 instruction layout ensures that PORT instructions are uniquely determined.

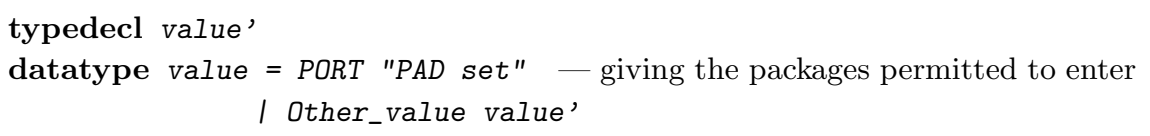

The abstract state itself is defined as a record. Each of the field names induces a corresponding selector function whose first argument is a value, typically called $s$, of type state.

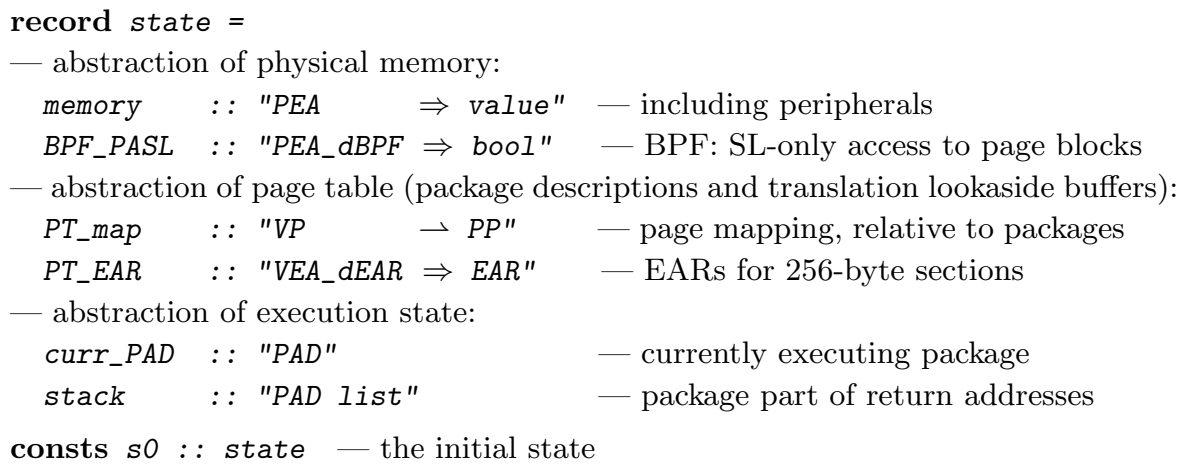


The state components BPF_PASL, PT_map, and PT_EAR each define a mapping only for the relevant sections of physical and virtual addresses, which helps to avoid redundancies in particular for update operations. Yet it is often convenient to perform the lookup operation with a full PEA or VEA, respectively. The auxiliary functions $B P_{-} P A S L, P E A$, and EAR, respectively, provide these liftings.

\subsection{Assumed Initial State Properties}

The security target [WN03] requires that all EARs should be initialized with a reasonable value. Since the exact value is immaterial for our analysis, we apply the standard technique, viz. to declare a constant giving the default EAR of memory sections without actually defining its value.

consts default_EAR :: "EAR" - underspecified

The functional specification requires that only the PSL package may call the SL package, which restricts the sets of packages within PORT instructions of SL. We specify this for the initial state so with the following axiom:

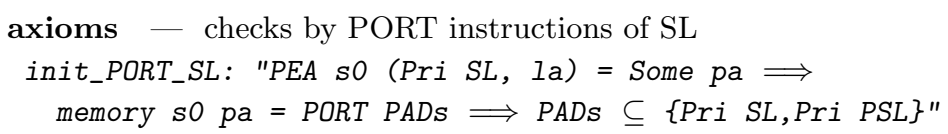

The axiom can be read as follows. For any VEA that belongs to SL (i.e., has the form (Pri SL, la) for some la), if in the initial state it is mapped to any PEA pa and a PORT instruction is stored at that address, then the associated set PADs of allowed packages may contain only SL and PSL.

A further important requirement is that the BPFs are reasonably set: for any physical pointer $p p$ and page block address, PASL should be true iff the page block is owned by SL, i.e. $p p$ is associated with some VEA belonging to SL:

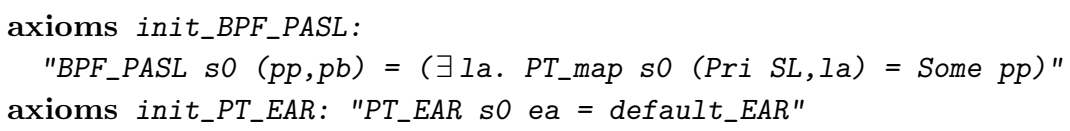

It is evident that the processor should start executing in the SL package with an empty return stack. Though we do not actually need these properties in our proofs, we state them for symmetry:

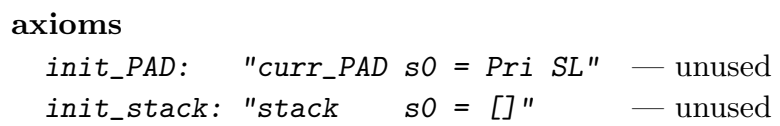




\subsection{Aliasing via Page Table}

By the construction of the page table mapping, there is the possibility that the mapping is non-injective, i.e., that multiple VEAs refer to the same PEA. The MMU device driver in the PSL package avoids such aliasing, but the page table may be manipulated directly by privileged packages in order to meet extraordinary needs for inter-package sharing. Our model is general enough to handle also such forms of aliasing. Naturally, in such cases the guarantees that can be made are weaker. In particular, conflicting EARs may arise, for example if a certain memory page is mapped for two different packages where one package sets the EAR such that all others should not be able to write to that memory page, while the other package claims to have write access by setting its EAR accordingly. We have identified a predicate on the page table contents that specifies conditions as weak as possible but still guaranteeing inter-package consistency of EARs: if two different packages $p$ and $p$ ' happen to map the same memory page then the EARs associated with that page should be both wW or both $R R$.

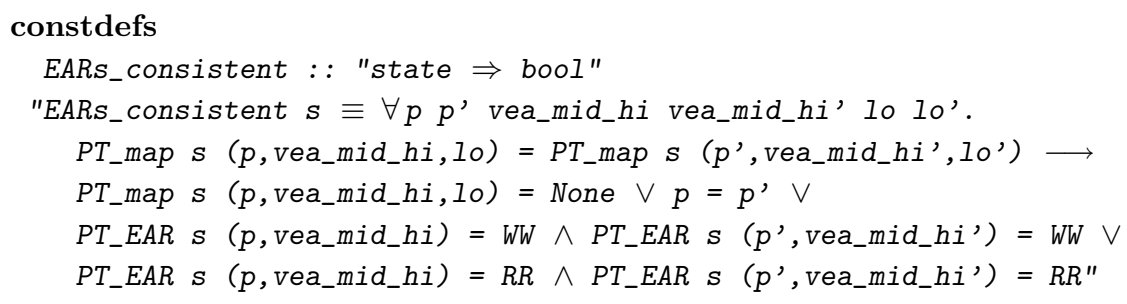

\subsection{Interface}

We define the SLE 88 memory management system as an Interacting State Machine (ISM) with a rather trivial interface: it has one input port named In and one output port named out.

datatype interface $=$ In $/$ Out

The messages exchanged with the environment are either instructions given to the system or results sent by the system. The instructions are abstractions of the usual CPU (micro-)instructions where we focus on code fetch (which is the first step of each instruction execution), memory read and write, various forms of branches, and write operations to various special registers including the page table. The chip may respond with positive or negative acknowledge or various traps (which will be explained where appropriate) in case of denied access.

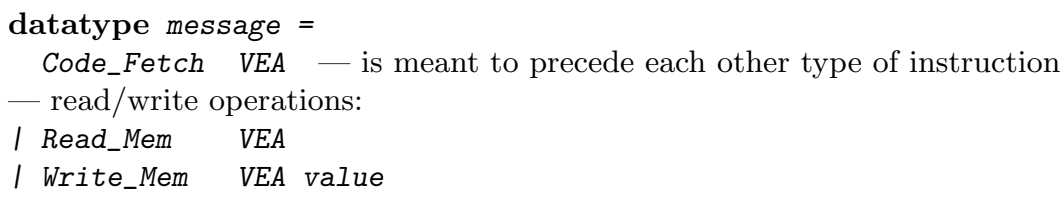


- control transfer operations:

I Jump VEA

I Call VEA

I Return

I Write_RetAddr VEA

- operations for setting security attributes and page table entries:

I Write_BPF_PASL PEA_dBPF bool

I Write_PT_EAR VEA_dEAR EAR

I Write_PT_map VP "PP option"

- outcome of operations:

I Ok I No - access granted or denied without generating a trap

| MPA | MPSF | RLCP | MPBF | PRIV | MCR - traps

\subsection{Auxiliary Access Functions}

For modeling the access control checks performed when executing access operations, it is beneficial to factor out common behavior and to reduce the complexity of the associated system transitions by defining dedicated auxiliary functions.

The function mem_access takes as its arguments the access mode, the current system state $s$, and the virtual address va to be accessed. It determines whether the current package, which is the subject (called source) of the operation at hand, is allowed to access - in the given mode - the package given by the PAD of va, which is the object of the operation (called target). In particular, it checks whether

- the virtual address is mapped to some existing PEA pa (and otherwise causes a Memory Protection Package Boundary Fault trap)

- the source is privileged and performs a read or write access where the target is some other package except SL ${ }^{10}$, or the EAR associated with va allows access with the given mode, making the distinction if the access is local or to some other package (and otherwise causes a Memory Protection Access Violation or MPBF trap)

- PASL is true for pa iff the target is SL (which checks consistency of the PASL setting), or SL accesses data - for testing purposes - in a page block not belonging to SL where PASL is true (and otherwise causes a Memory Protection Security Field trap).

It is defined as

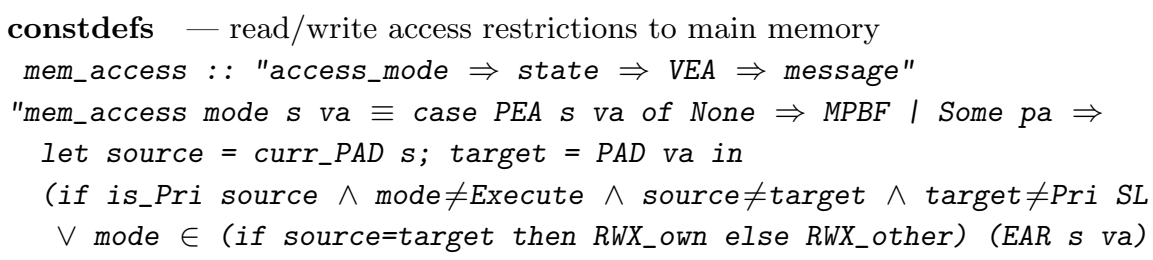

10 This operation is typical for e.g. the operating system loading a package 


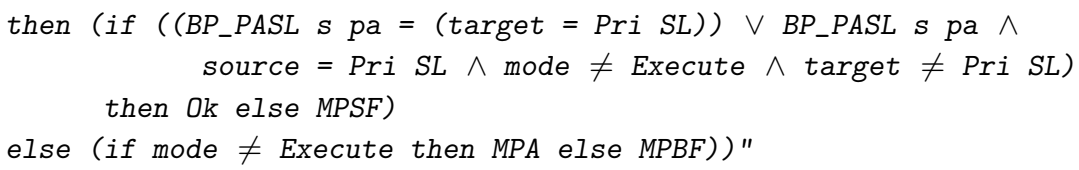

The function Call_access takes as its arguments the current system state $s$ and the virtual address va to be called. It grants intra-package calls (i.e., the PAD of the target va equals the current PAD), and otherwise checks whether

- va is mapped to some PEA pa (and otherwise causes a Memory Protection Package Boundary Fault trap)

- the value stored at pa is a PORT instruction (and otherwise causes a Privileged Instruction trap)

- the PORT instruction allows the current package to enter (and otherwise typically just returns from the call without causing a trap).

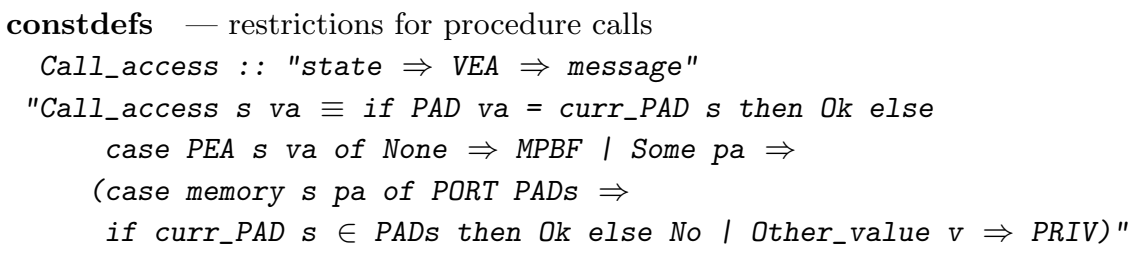

The function Write_PT_access takes as its arguments the current system state $s$ and the package to be affected. Writing to the page table is granted only if the current package is privileged. It must be even SL if the target package is SL. Otherwise a Memory Protection Core Register Address trap is generated.

constdefs - restrictions for writing page table information

Write_PT_access : : "state $\Rightarrow P A D \Rightarrow$ message"

"Write_PT_access $s$ target $\equiv$ if(target=Pri $S L \longrightarrow$ curr_PAD s=Pri SL) $\wedge$ is_Pri (curr_PAD s) then Ok else MCR"

\subsection{Transitions}

The core of our security model is the definition of the ISM that specifies the overall memory management system of the SLE 88. The definition follows the format given in $§ 3.3$. For each kind of instruction that may be issued (by sending it to the ISM) there is one transition rule. Transitions are atomic and instruction execution is meant to be sequential. The system reacts by outputting a value that indicates granted or denied access, where the latter typically leads to a trap. In our abstract model there is no need to specify trap handling. A couple of the transition rules have preconditions, and most of them have postconditions specifying changes to the system state. Since conditional changes to mappings are very common, we define the syntactic abbreviation "c ? $f(x:=y) " \rightarrow$ "if $c$ then $f(x:=y)$ else $f$ ". 


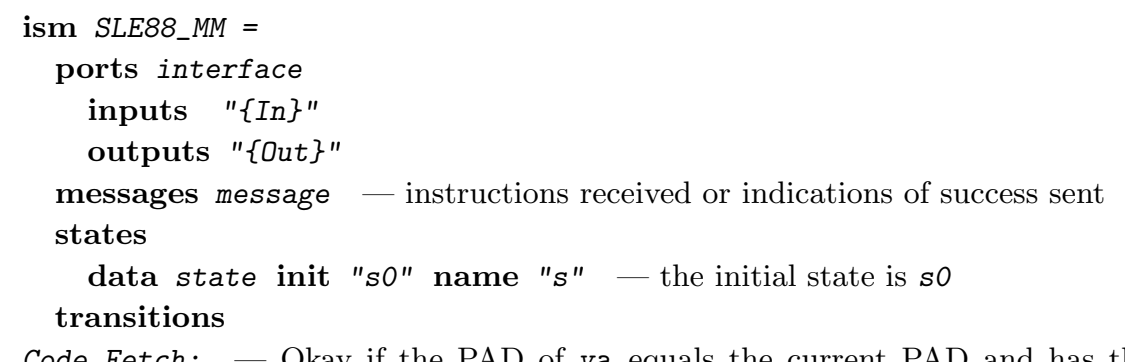

Code_Fetch: - Okay if the PAD of va equals the current PAD and has the EAR $X$ - and va is mapped to some page block where PASL is true iff the current $\mathrm{PAD}$ is $\mathrm{SL}$.

in In "[Code_Fetch va]"

out Out "[mem_access Execute s va]"

Read_Mem:

in In "[Read_Mem va]"

out Out "[mem_access Read s va]"

Write_Mem: - Sets the memory cell at address va to the value $v$ by the value $v$ if access is granted. If the target package is SL and PASL is false for the affected page block, it may non-deterministically - as specified using the free variable belated_MPSF - write the value even though the access is denied, namely if the MPSF trap is delayed.

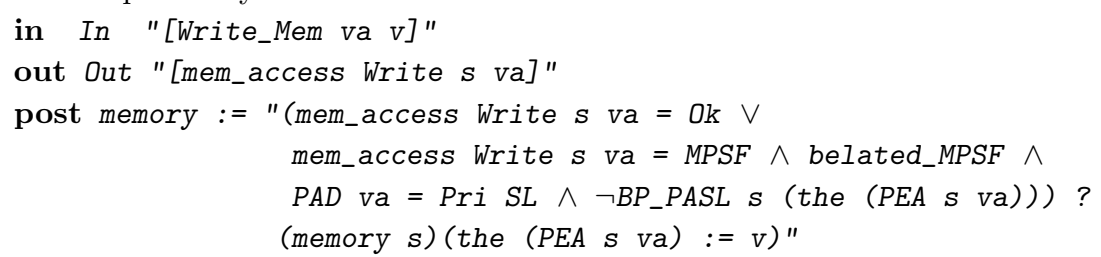

Call: - If the call is allowed then the current PAD is updated and its old value is pushed on the abstract return stack.

in In "[Call va]"

out Out "[Call_access s va]"

post curr_PAD:="if Call_access $s$ va=Ok then $P A D$ va else curr_PAD s", stack :="if Call_access $s$ va=Ok then curr_PAD s\#stack $s$

else stack s"

Return: - The first precondition states that the stack is non-empty with top element $r$ while the second precondition just gives an abbreviation. A return into SL is not allowed, causing a Return Leave Current Package Mistake trap, otherwise $r$ is popped from the stack and becomes the new current PAD.

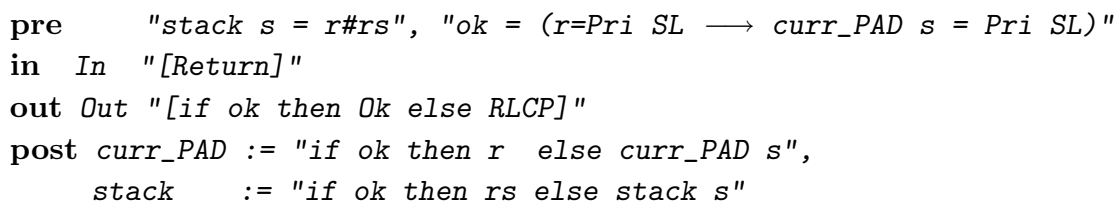


Write_RetAddr: - Setting the return address, i.e. the stack top, to an address whose PAD is different from the current one is possible only for privileged packages.

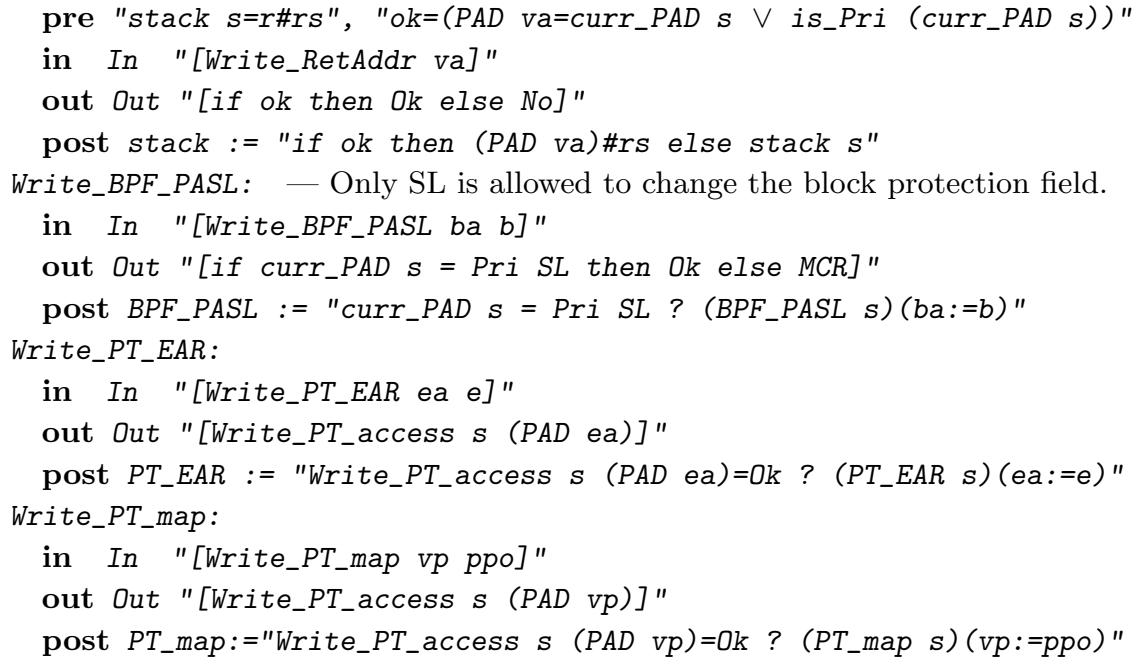

Having given all the above definitions, we use them for stating and proving security properties. Many of these require additional assumptions on reasonable behavior of the SL package, which we will give as additional axioms restricting the transitions of the ISM.

\section{Security Properties}

\subsection{Overview}

Given the system model in the form of an ISM, we are ready to formalize the security requirements of $\S 2.3$ as properties of (sequences of) ISM state transitions. Since the security requirements are formulated on a very high level, expressing the properties and arguing for their completeness has been appropriately done by discussing them with the requirement engineers, taking into account the SLE 88 specifications and the justifications given in the security target, which define details like access modes, EARs, the PASL attribute, and their intended effect.

The main concern of the security requirements is separation of applications, i.e., suitable restriction of inter-package access, which we address by the theorems

- interpackage_Read_Mem_respects_EAR, interpackage_Write_Mem_respects_EAR, and Code_Fetch_only_Iocal_X addressing inter-package read/write/execute protection, described in $\S 5.2$, and

- interpackage_transfer_only_via_Call_to_suitable_PORT_or_Return, addressing inter-package control transfer, described in $\$ 5.4$. 
Another critical issue is special protection of the SL package because it manages the security attributes, onto which access control is based. By stating the series of theorems given in $§ 5.3$ culminating in only_SL_changes_SL_memory and only_SL_reads_SL_memory, and in $\S 5.4$ culminating in only_PSL_enters_SL, we have covered all properties implied by the security requirements.

Proving that the theorems hold for the given system model completes the formal security analysis. The proofs show some inherent complexity, for instance by having to consider layered protection mechanisms and effects of aliasing, i.e., non-injective page table mappings. Still, due to adequate modeling and the powerful Isabelle proof system, developing the machinechecked proofs has been a matter of just a few days.

The act of conducting proofs identifies necessary assumptions concerning the initial state and the access control attribute settings for the SL package. In particular, we introduce a notion of consistency of EAR assignments that is useful in case of aliasing.

\subsection{Inter-package Access Protection}

Our first two theorems state basic properties of inter-package read and write access. If in any state $s$, a read instruction for some virtual address va not belonging to the current package is successful, then this has been done by a privileged package accessing a package other than SL, or read (or read/write) access is granted by the EAR associated with va. Note that the access rights are determined at the virtual (not: physical) address level, which opens up the possibility of inconsistencies incurred by aliasing, i.e. different access paths to the same physical memory area. In effect, the accessibility of a memory area is determined by the minimum protection of all related EARs. Only if inter-package consistency of the EARs is ensured, we can guarantee that for any other virtual address va' belonging to a different package and mapped to the same physical address, the associated EAR is the same (and cannot be WR because this EAR is not symmetric) and thus no unwanted access is possible.

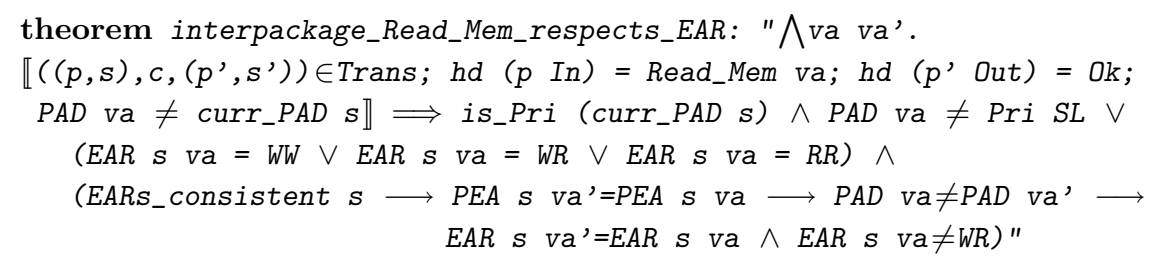

Some notational remarks are advisable here: in Isabelle formulas, ' $\wedge$ ' is a universal quantifier; multiple premises are bracketed using ' $\llbracket$ ' and ' $\rrbracket$ ' and separated using ';'. The term hd ( $p$ In) refers to the input and hd ( $p$ ' Out) to the output of the transition $\left((p, s), c,\left(p^{\prime}, s^{\prime}\right)\right) \in$ Trans which takes the state $s$ to $s^{\prime}$ and corresponds to $\left((p, s),\left(p^{\prime}, s^{\prime}\right)\right) \in \operatorname{Trans}(a)$ as defined in $\S 3.2 .3$. The free variable $c$ can be ignored. Logical conjunction ' $\wedge$ ' has higher syntactic precedence than disjunction ' $V$ ' and implication ' $\longrightarrow$ '. 
The proof of this theorem proceeds by case distinction on the transition rules. The only non-trivial case is the one of Read_Mem where we unfold the definitions of mem_access, RWX_other, and EARs_consistent and perform standard predicate-logical reasoning and term rewriting. We reproduce the actual proof script for this theorem in order to give an impression of how the Isabelle proofs (in the traditional tactic style) actually look like. The execution of such a script typically takes a few seconds.

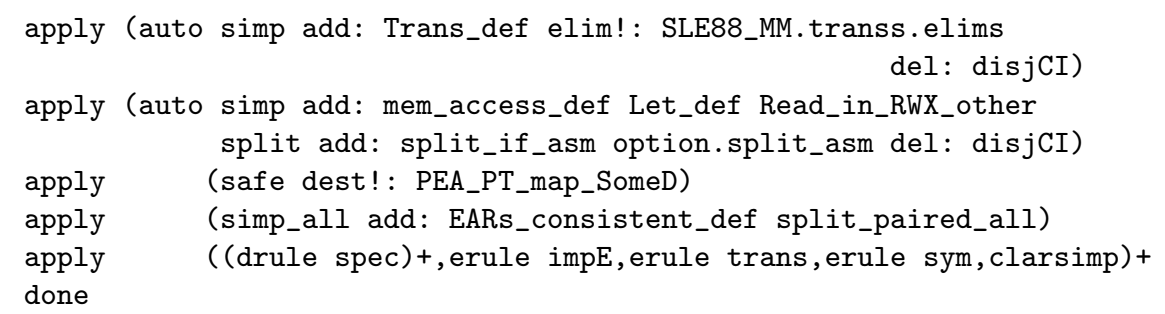

The analogous theorem concerning the write instruction is a bit simpler because there are less cases that allow write access:

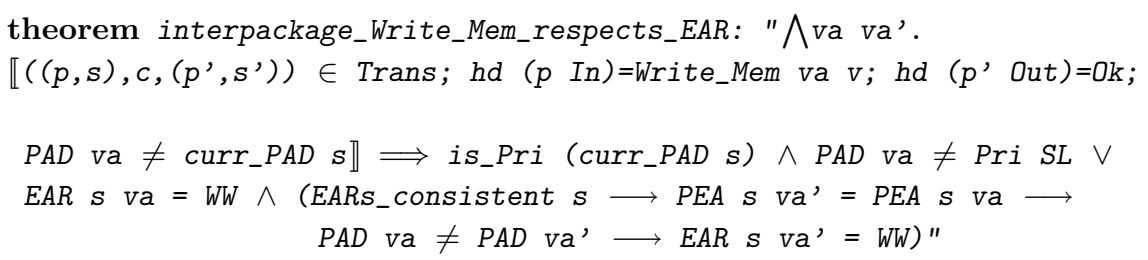

As can be derived easily from the transition rule for the Code_Fetch operation and the definition of mem_access, code may be executed only from memory that belongs to the current package and that is marked with the EAR $X$-:

theorem Code_Fetch_only_local_X:

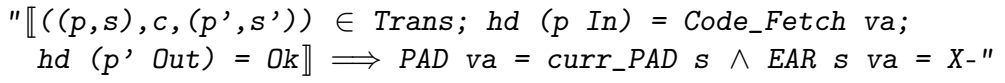

\section{3 Read/Write Protection for SL Memory}

The next bunch of lemmas and theorems focus on the protection of the memory areas of the SL package. Typically they involve transitions reachable within a open run of the processor, i.e., elements of a sequence $t s \in$ TRuns where TRuns corresponds to Runs $(a)$ as defined in $§ 3.2 .4$.

Only SL may change the mapping of PEAs belonging to SL. More precisely, for any state transition from $s$ to $s$ ' within a run, unless the current package is SL, the page table mapping concerning SL is the same for $s$ and $s^{\prime}$. This is a simple consequence of the definition of Write_ $P T_{-}$access used in the rule Write_PT_map.

theorem only_SL_changes_PT_map_of_SL:

$" \llbracket t s \in$ TRuns; $\left((p, s), c,\left(p^{\prime}, s^{\prime}\right)\right) \in$ set $t s ; c_{c u r r} P A D s \neq \operatorname{Pri} s L \rrbracket \Longrightarrow$

$P T_{-}$map $s(\operatorname{Pri} S L, I v p)=P T_{-}$map $s^{\prime}(\operatorname{Pri} S L, I v p) "$ 
The analogous property holds for the EARs associated with SL memory:

theorem only_SL_changes_EAR_of_SL:

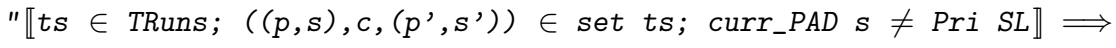

EAR s (Pri SL, Iva) =EAR s' (Pri SL, Iva)"

Similarly, only privileged packages may change EARs:

theorem only_Pri_change_EAR:

$" \llbracket t s \in$ TRuns; $\left((p, s), c,\left(p^{\prime}, s^{\prime}\right)\right) \in \operatorname{set} t s ; \neg i s_{-} P r i \quad\left(\operatorname{curr}_{-} P A D s\right) \rrbracket \Longrightarrow$

$E A R \mathrm{~s}$ va = EAR s' va"

Later we will need an invariant stating that the EARs associated with SL deny any access by other packages. In order to establish this property, we have to assume that the default EAR denies such access as well and that SL sticks to this policy when writing EARs:

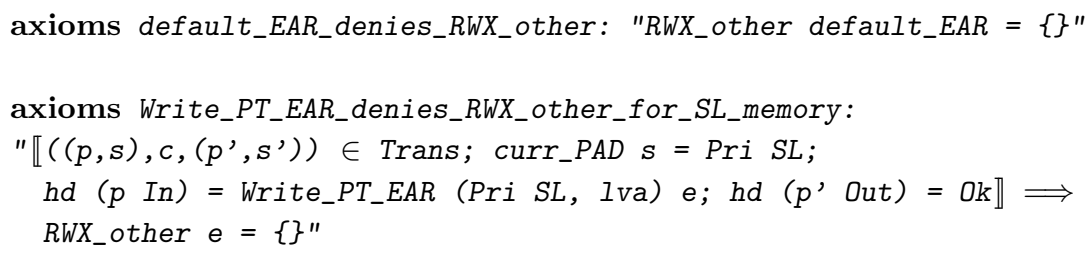

The necessity of such axioms makes explicit some important assumptions on the initialization of security attributes and the behavior of SL and therefore gives valuable feedback for system software development.

With the help of the two axioms just given and the axiom init_PT_EAR given in $\S 4.5$, we can prove the invariant easily by induction on the length of transition sequences. In terms of the Isabelle/HOL implementation of ISMs, this invariant can be expressed in the following compact way:

lemma $S L_{-}$pages_deny_RWX_other:

"Inv $(\lambda s . \forall$ Iva. RWX_other $(E A R s(\operatorname{Pri} S L, I v a))=\{\}) "$

It reads as follows. For any reachable state $s$ and any virtual address within the SL package, the associated EARs for other packages is the empty set. Similar comments apply to the invariant that PASL is true for all memory belonging to SL. It requires the additional assumptions that SL writes the block protection fields and the page table entries for its memory only in a way such that PASL remains true:

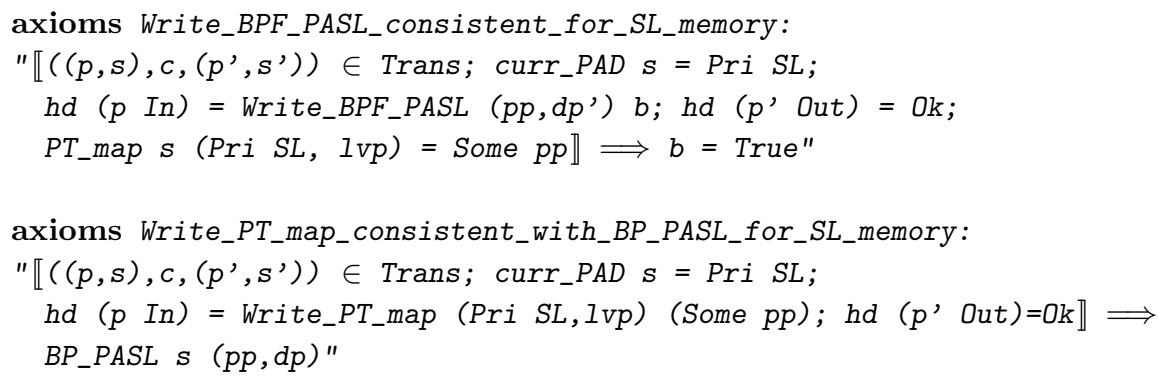


Together with the axiom init_BPF_PASL also given in $\S 4.5$, we can prove the invariant in an analogous way.

lemma SL_memory_has_PASL:

"Inv ( $\lambda \mathrm{s} . \forall$ Iva pa dp. PEA s (Pri $S L, I v a)=$ Some pa $\longrightarrow$ BP_PASL s pa)"

Taking advantage of the two invariance lemmas just given, we prove that only SL can change memory allocated to SL. The proof uses the invariant SL_memory_has_PASL concerning PASL three times, where in all these cases there is aliasing in the page table such that the same physical memory area is allocated to both SL and some non-SL package. Thus we can conclude that PASL plays an important role for detecting such (unwanted) aliasing wrt. SL memory.

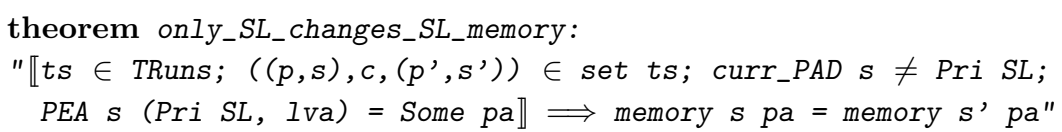

The theorem stating that only SL can read memory allocated to SL requires only the invariant $S L_{-}$pages_deny_RWX_other concerning EARs of SL:

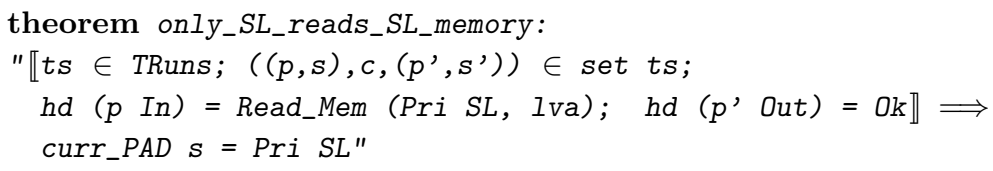

\subsection{Inter-package Control Transfer and PORT Instructions}

Given theorem Code_Fetch_only_local_X, the only form of inter-package code access that needs to be addressed further is transfer of control where the current package changes.

Our next theorem states that the only possibilities for such control transfer is a legal procedure call or return; in more detail: if there is a transition from state $s$ to $s$ ' where the current package changes, then either it has been caused by a call whose target is a virtual address va mapped to a physical address pa containing a PORT instruction that explicitly allows the calling package to enter, or it has been caused by a return to a package other than SL:

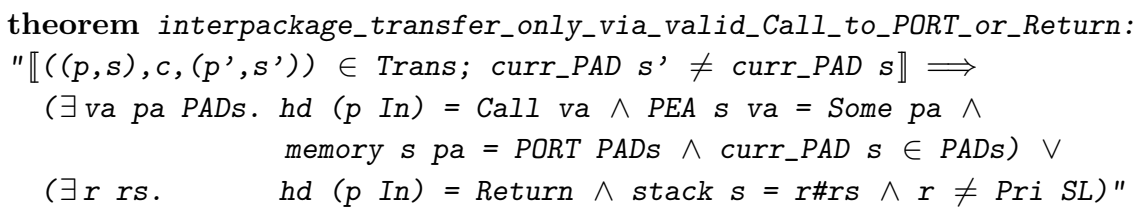

The proof of this theorem is straightforward by case distinction on all instructions available and unfolding the definition of Call_access.

Much more involved is the proof of our final theorem stating that only PSL can enter SL: we need an invariant that all PORT instructions contained in memory allocated to SL allow only calls by SL itself and by PSL. 
This in turn requires two assumptions that SL writes memory allocated to itself and the page table entries for its memory only in a way such that the invariant is maintained:

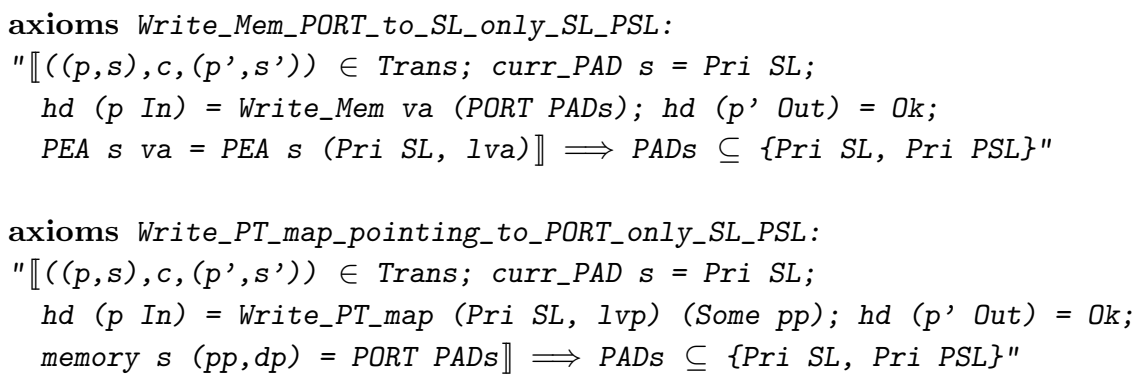

Note the essential role of aliasing in the first of these axioms: the instruction intended to write a PORT instruction at virtual address va might affect SL memory even if va does not belong to SL, namely if there is some other virtual address (Pri SL, Iva) that happens to be mapped to the same physical address.

Apart from the two axioms, the proof of the invariant requires the axiom init_PORT_SL given in $\S 4.5$ as well as the theorems only_SL_changes_SL_memory and only_SL_changes_PT_map_of_SL.

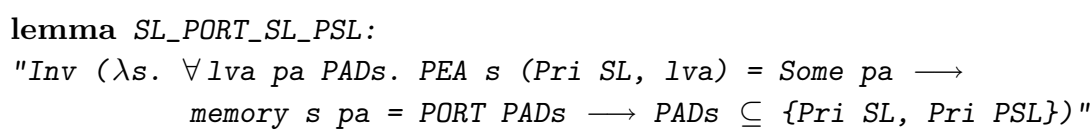

Exploiting the invariant, the theorem can be proven in just a few steps. It reads as follows: for any sequence of transitions ts that may result from a system run and any state transition from $s$ to $s^{\prime}$ within it, if SL becomes the current package in $s^{\prime}$, the current package of the pre-state $s$ must have been PSL.

theorem only_PSL_enters_SL:

$" \llbracket t s \in$ TRuns; $\left((p, s), c,\left(p^{\prime}, s^{\prime}\right)\right) \in$ set $t s ; \operatorname{curr}_{-} P A D s \neq$ Pri $S L$;

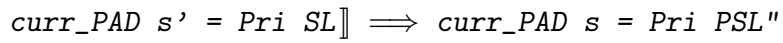

This finishes our abstract formal analysis of the SLE88 memory management.

\section{Conclusion}

We have introduced a security model for the memory management of the SLE88 smart card processor chip. Memory management contributes to security by providing access control mechanisms on the levels of both virtual and physical memory addresses, allowing to separate applications and privileged SW packages (e.g., the operating system and the security layer SL) as well as applications from each other. Access control is guided by a policy comprising both discretionary (by effective access rights EAR) and mandatory 
(wrt. SL and privileged packages) rules. Enforcing the policy is non-trivial: the ability to change EARs and address mappings, the interacting levels of protection, aliasing in address translation, inter-package calls, and the peculiarities of SL have to be considered.

The model gives an abstract view of the SLE8 8 by concentrating on memory access and its protection only, leaving out details of the system and application functionality. Abstraction is achieved by reductions on the data structure and interface design and by underspecification. For instance, many data types used in the model are declared but not actually defined.

Carrying out the formal modeling work turned out to be worthwhile, because it provided new insights and lead to clarification of so far fuzzy concepts. Formal reasoning resulted in a minimal set of requirements on non-injective address mappings that guarantee the maintenance of the security properties. These requirements are given by restrictions on admissible combinations of EAR settings. The derived notion of EAR consistency is the least restrictive one preserving security and offers much more flexibility compared to simply forbidding aliasing. Formal analysis showed that security depends on assumptions on the initial state (e.g., initial EAR and PASL settings) as well as on benign behavior of SL. The assumptions can be interpreted as requirements on configuration upon delivery and on the software development of privileged packages. They clearly indicate the distribution of responsibility between the Target of Evaluation and its environment. Last, but not least, formal arguments lead to a clarification of the role of PASL: the PASL mechanism does not provide additional protection in case of weak EARs for SL, but protects against effects resulting from undesired mapping of both SL and non-SL virtual addresses to the same physical address.

To summarize, results of the modeling and proving process are the identification of relevant assumptions on the system environment and the derivation of new insights in the memory management and its security properties. The cost-benefit ratio is adequate: the whole work required no more than a six weeks effort, largely due to the availability of adequate tool support through Isabelle/HOL and the ISM approach. Thus, the SLE88 memory management security model is an excellent example for the value of formal security modeling in practical industrial-scale applications.

\section{References}

AHIP01. Atmel, Hitachi Europe, Infineon Technologies, and Philips Semiconductors. Smartcard IC Platform Protection Profile, Version 1.0, July 2001. http://www.bsi.de/cc/pplist/ssvgpp01.pdf.

AHIP02. Atmel, Hitachi Europe, Infineon Technologies, and Philips Semiconductors. Smartcard Integrated Circuit Platform Augmentations, Version 1.0, March 2002. http://www.bsi.de/cc/pplist/augpp002.pdf.

Bib77. K.J. Biba. Integrity considerations for secure computer systems. Technical Report MTR 3153, Mitre Corporation, Bedford MA, 1977. 
BL73. D.E. Bell and L. LaPadula. Secure Computer Systems: Mathematical Foundations (NTIS AD-770 768), A Mathematical Model (NTIS AD771 543), A Refinement of the Mathematical Model (NTIS AD-780 528). Technical Report MTR 2547, Mitre Corporation, Bedford MA, 1973.

CC99. Common Criteria for Information Technology Security Evaluation (CC), Version 2.1, 1999. ISO/IEC 15408.

CW87. D.R. Clark and D.R. Wilson. A comparison of commercial and military computer security policies. In Symposium on Security and Privacy, pages 184-194. IEEE Computer Society Press, 1987.

GM82. J. A. Goguen and J. Meseguer. Security policies and security models. In Symposium on Security and Privacy. IEEE Computer Society Press, 1982.

HSSS96. Franz Huber, Bernhard Schätz, Alexander Schmidt, and Katharina Spies. Autofocus - a tool for distributed systems specification. In Proceedings FTRTFT'96 - Formal Techniques in Real-Time and FaultTolerant Systems, volume 1135 of $L N C S$, pages 467-470. SpringerVerlag, 1996. See also http://autofocus.in.tum.de/index-e.html.

KO03. Thomas Kuhn and David von Oheimb. Interacting State Machines for mobility. In K. Araki, S. Gnesi, and D. Mandrioli, editors, Proc. of the $12^{\text {th }}$ International FME Symposium (FM'03), volume 2805 of LNCS. Springer, September 2003. http://ddvo.net/papers/ISMfM.html.

LKW00. Volkmar Lotz, Volker Kessler, and Georg Walter. A Formal Security Model for Microprocessor Hardware. In IEEE Transactions on Software Engineering, volume 26, pages 702-712, August 2000.

LT89. Nancy Lynch and Mark Tuttle. An introduction to input/output automata. CWI Quarterly, 2(3):219-246, 1989. http://theory.lcs.mit. edu/tds/papers/Lynch/CWI89.html.

MT00. Stephanie Motre and Corinne Teri. Using B method to formalize the Java Card runtime security policy for a Common Criteria evaluation. In $23^{\text {rd }}$ National Information Systems Security Conference, 2000. http: //csrc.nist.gov/nissc/2000/proceedings/toc.html.

Nan02. Sebastian Nanz. Integration of CASE tools and theorem provers: a framework for system modeling and verification with AutoFocus and Isabelle. Master's thesis, TU München, 2002. http://www.doc.ic.ac. uk/ nanz/publications/csthesis/.

NPW02. Tobias Nipkow, Lawrence Paulson, and Markus Wenzel. Isabelle/HOL - A Proof Assistant for Higher-Order Logic, volume 2283 of LNCS. Springer, 2002. See also http://isabelle.in.tum.de/docs.html.

Ohe02. David von Oheimb. Interacting State Machines: a stateful approach to proving security. In Ali E. Abdallah, Peter Ryan, and Steve Schneider, editors, Formal Aspects of Security, volume 2629 of LNCS, pages 15-32. Springer-Verlag, 2002. http://ddvo.net/papers/ISMs.html.

Ohe04. David von Oheimb. Information flow control revisited: Noninfluence $=$ Noninterference + Nonleakage. In Proc. of the $9^{\text {th }}$ European Symposium on Research in Computer Security, LNCS. Springer, 2004. http:// ddvo.net/papers/Noninfluence.html.

OL02. David von Oheimb and Volkmar Lotz. Formal Security Analysis with Interacting State Machines. In Dieter Gollmann, Günter Karjoth, and Michael Waidner, editors, Proc. of the $7^{\text {th }}$ European Symposium on Research in Computer Security (ESORICS), volume 2502 of LNCS, pages 212-228. Springer, 2002. http://ddvo.net/papers/FSA_ISM.html. 
OL03. David von Oheimb and Volkmar Lotz. Generic Interacting State Machines and their instantiation with dynamic features. In Jin Song Dong and Jim Woodcock, editors, Formal Methods and Software Engineering (ICFEM), volume 2885 of LNCS, pages 144-166. Springer, November 2003. http://ddvo.net/papers/GenISMs.html.

ON02. David von Oheimb and Sebastian Nanz. ISM Homepage: Documentation, sources and distribution, 2002. http://ddvo.net/ISM/.

Rus92. John Rushby. Noninterference, Transitivity, and Channel-Control Security Policies. Technical Report CS-92-02, SRI International, 1992.

$\mathrm{SRS}^{+}$00. G. Schellhorn, W. Reif, A. Schairer, P. Karger, V Austel, and D. Toll. Verification of a formal security model for multiapplicative smart cards. In Frédéric Cuppens, Yves Deswarte, Dieter Gollmann, and Michael Waidner, editors, Proc. of the $6^{\text {th }}$ European Symposium on Research in Computer Security (ESORICS), volume 1895 of LNCS. Springer, 2000.

WN03. Georg Walter and Jürgen Noller, Infineon Technologies. SLE88CX720P / m1491 Security Target. BSI, Version 1.00, March 2003. 